\title{
Transconjunctival Blepharoptosis Surgery: A Review of Posterior Approach Ptosis Surgery and Posterior Approach White-Line Advancement
}

\author{
Vikesh Patel and Raman Malhotra*
}

\author{
CorneoPlastic Unit, Queen Victoria Hospital, East Grinstead, RH19 3DZ, UK
}

\begin{abstract}
Posterior approach blepharoptosis surgery, via the transconjunctival route, was probably the first method of surgery employed to shorten the levator palpebrae superioris (LPS) muscle. A review of the literature demonstrates how surgery has evolved since Blaskovics' first described his technique in 1923. We describe our newer method of posterior approach white-line advancement blepharoptosis repair which is now an option in the majority of aponeurotic ptosis with moderate to good levator function.
\end{abstract}

Keywords: Transconjunctival Blepharoptosis Surgery, Ptosis Surgery, White-Line Advancement, Müller's muscle conjunctival resection.

\section{HISTORY OF TRANSCONJUNCTIVAL BLEPHARO- PTOSIS SURGERY}

Posterior approach blepharoptosis surgery, via the transconjunctival route, was probably the first method of surgery employed to shorten the levator muscle [1-5]. In 1923 Blaskovics [1,2] first described his technique of extensive dissection of the levator muscle from its surrounding structures prior to its resection. Two sets of sutures were used, the first three were through the levator aponeurosis and the cut edge of the conjunctiva and the second three were fold-forming sutures through the levator aponeurosis only, and then both were passed anteriorly through the tarsus and skin. This was combined with a tarsectomy in all cases.

Posterior approach surgery was further addressed by Agatston [3] when he stated in 1942 that this technique was becomingly increasingly popular in the United States. In 1953 Berke [4], operating mainly on congenital cases, further simplified Blaskovics technique, by using only one set of sutures and no tarsal excision was carried out. Following the early papers demonstrating the technique of anterior approach ptosis repair, posterior approach surgery was preferred when focussing on the posterior lamellar structures; tarsus, conjunctiva and Müller's muscle.

Müller's muscle conjunctival resection (MMCR) is traditionally performed in patients with mild ptosis who show eyelid elevation following instillation of topical phenylephrine. In 1961 Fasanella and Servat [6] described a procedure for patients with minimal blepharoptosis in which they applied two curved haemostats to the everted superior tarsus, ran a suture above them, and excised the tissues held by them. They initially described a "resection of Müller's muscle, levator, tarsus and conjunctiva", however histological studies have since shown that tissue resected

\footnotetext{
*Address correspondence to this author at the Corneoplastic Unit, Queen Victoria Hospital, Holtye Road, East Grinstead, West Sussex, RH19 3DZ, UK; Tel: 01342 414549; Fax: 01342 414106;

E-mail: raman.malhotra@qvh.nhs.uk
}

consisted of a predominately tarsoconjunctival layer [7, 8]. We now regard the Fasanella-Servat procedure, which involves a blind approach, as a predominately tarsoconjunctival resection.

In 1975 Putterman and Urist [9] described their technique of MMCR. After separating the Müller's and conjunctiva from the surrounding structures a modified clamp replaced the curved haemostats and the tissue was excised. There was no resection of the tarsus. They found that in most patients the upper eyelid was elevated to a normal level following instillation of $10 \%$ phenylephrine and an $8 \mathrm{~mm}$ MMCR produced a similar result. The authors recommended a $9 \mathrm{~mm}$ resection if the phenylephrine test demonstrated a lower eyelid reponse, or a $7 \mathrm{~mm}$ resection if the response was higher than desired.

At the same time, in Europe, a technique for Müller's muscle resection was being popularized by Abraham Werb in our unit. This involved an incision at the superior border of the tarsal plate, dissection and excision of a conjunctivalMüller flap and advancement of the white-line (distal end of the thick anterior levator aponeurosis) to the tarsal plate and through to the skin crease. To our knowledge, Mr Werb was first to emphasise the importance of advancing the white-line in this technique.

Subsequent modifications to the MMCR technique describe a wide variety of algorithms to determine the appropriate amount of tissue resection to correct a given degree of ptosis [10, 11], an open sky MMCR and a conjunctival sparing Müller resection $[12,13]$. More recently we have described a surgical technique involving advancement of the white-line via a transconjunctival posterior approach without resection of Müller's muscle.

It appears that widely disparate amounts of resection may yield acceptable surgical results for similar degrees of ptosis. Buchman et al. [14] analysed histological specimens from 40 Fasanella-Servat cases which revealed $88 \%$ of cases had absent to minimal smooth muscle resection. However these patients had equally successful results in comparison to 
patients with moderate or large amounts of smooth muscle resection

Dresner [11] describes a further modification of the MMCR technique whereby the amount of resection depends on the response to phenylephrine testing. When phenylephrine testing results in at least $2 \mathrm{~mm}$ of eyelid elevation, Dresner applied the following algorithm: $4 \mathrm{~mm}$ of resection for $1 \mathrm{~mm}$ of ptosis, $6 \mathrm{~mm}$ of resection for $1.5 \mathrm{~mm}$ of ptosis, $10 \mathrm{~mm}$ of resection for $2 \mathrm{~mm}$ of ptosis, and 11 or $12 \mathrm{~mm}$ of resection for $>3 \mathrm{~mm}$ of ptosis. Weinstein and Berger [10] suggest that a linear relation may exist between the resultant eyelid elevation and quantity of Müller's muscle resection. This technique begins with $8 \mathrm{~mm}$ of resection to correct 2 $\mathrm{mm}$ of ptosis, then adds or subtracts $1 \mathrm{~mm}$ of resection to affect the final eyelid position by $0.25 \mathrm{~mm}$. Perry et al. [15] devised a new algorithm based on this theory whereby $9 \mathrm{~mm}$ of Müller's resection should result in the same elevation that is produced by maximal stimulation with phenylephrine. If phenylephrine testing results in an undercorrection then tarsus was added to the resection.

More recently Lake et al. [12] and Baldwin et al. [16] reported their series of open sky MMCR in both phenylephrine test positive and negative groups. Their technique allowed direct visualization of Müller's muscle before its subtotal resection. Double-armed sutures were passed through the Müller's muscle stump and the upper border of the tarsus through to the skin crease. As the sutures are passed through the stump of Müller's muscle and then through the tarsus, one is in effect advancing the levator aponeuorosis and attaching it to the tarsal plate and skin crease. They suggested that the success with the procedure lay, not from the degree of resection of Müller's muscle, but from the consequent advancement of the levator aponeurosis. This theory was further supported by the success of the procedure in phenylephrine negative patients [16]. More recently a conjunctival sparing technique has been reported as well [13].

\section{ANATOMICAL REASONS FOR THE SUCCESS OF MÜLLER'S MUSCLE CONJUNCTIVAL RESECTION}

The anatomical reasons for the success of Müller's muscle conjunctival resection has been a matter of debate for some time now. Several mechanisms have been suggested for the success of this technique, which include vertical posterior lamellar shortening, secondary contractile cicatrisation of the wound, or advancement of the Müller's muscle-levator aponeurosis complex on the tarsus $[11,17$, 18]. Increasingly, it has been felt that the success of the procedure is due to advancement of the levator muscle itself, along with the aponeurosis. The mechanism by which Müller's muscle resection alleviates ptosis would therefore be by transmitting the contraction force of the levator muscle directly to the tarsal plate instead of through its aponeurotic attachment.

\section{TECHNIQUE OF POSTERIOR APPROACH WHITE LINE ADVANCEMENT}

Advancement of a dehisced levator aponeurosis, as described in our technique, combines achievement of a predictable post-operative height expected with aponeurosis re-attachment and a predictable normal-looking eyelid contour seen with posterior-approach Müller's muscle resection surgery, yet avoids the need to excise any tissue, namely Müller's muscle or conjunctiva.

The procedure is usually performed under local anaesthesia with subcutaneous infiltration, both along the skin crease and in the mid-pupil pretarsal region and $0.5 \mathrm{ml}$ subconjunctival infiltration upon eyelid eversion using $2 \%$ lignocaine with 1:80000 adrenaline. The desired skin crease is marked and a 4-0 silk traction suture placed in the grey line of the upper eyelid, which is then everted over a Desmarres retractor. Gentle diathermy is applied prior to a

conjunctival incision with a no 15 Bard parker blade along but above the superior border of the tarsus. Müller's muscle and conjunctiva is dissected off as a composite flap until the white-line is identified. A double armed 5-0 vicryl ${ }^{\mathbb{B}}$ suture is placed centrally through the posterior belly of the white-line (Fig. 1A), in a forehand manner and is then passed through the conjunctival surface of the tarsal plate, $1 \mathrm{~mm}$ below its superior border (Fig. 1B), and then through to the skin. Gentle diathermy and scraping away of the epithelial surface of the superior border of the tarsal plate prior to passing the suture may be performed in order to provide a better more reliable long term adhesion between the white-line and the tarsus. The suture is captured through the skin in the region of the skin crease. The eyelid height and contour is assessed after tying this first suture in a bow (Fig. 1C) and care is taken to ensure there is no slippage of the suture. If the eyelid position is deemed to be satisfactory, the suture is relaxed and a second suture is placed within 2-3 mm lateral to the first in the method described above. Both sutures are then tied. If the eyelid height is too low after the first suture, it can then be relaxed and a second suture passed higher through the white-line and again through the tarsal plate and skin. If the upper eyelid contour appears peaked after the first suture, then this is relaxed and a second suture placed more central to the location of the peak. Using this method of altering the position of the second suture enables minor adjustments to eyelid height and contour without the undue delay of removing the first suture in the majority of cases. In such situations, the first suture is gently tied to act as a "support" rather than a "cardinal" suture. In the postoperative period the absorbable sutures are not removed and left to dissolve spontaneously. Both Müller's muscle and conjunctiva are left to heal spontaneously with no excision of these structures. In those cases with significant dermatochalasis the procedure can be combined with a blepharoplasty. This is carried out prior to eyelid eversion and white-line advancement.

\section{TIPS FOR POSTERIOR APPROACH WHITE-LINE ADVANCEMENT}

It is important that the sutures are placed into the healthier white sheet. We know from previous studies [19, 20] that the levator aponeurosis particularly in Asian eyelids comprises two layers, of which the anterior layer is thick with less smooth muscle fibres, and reflects superiorly a few millimeters above the tarsus to become contiguous with the orbital septum. The posterior layer is thin with more smooth 
(A)

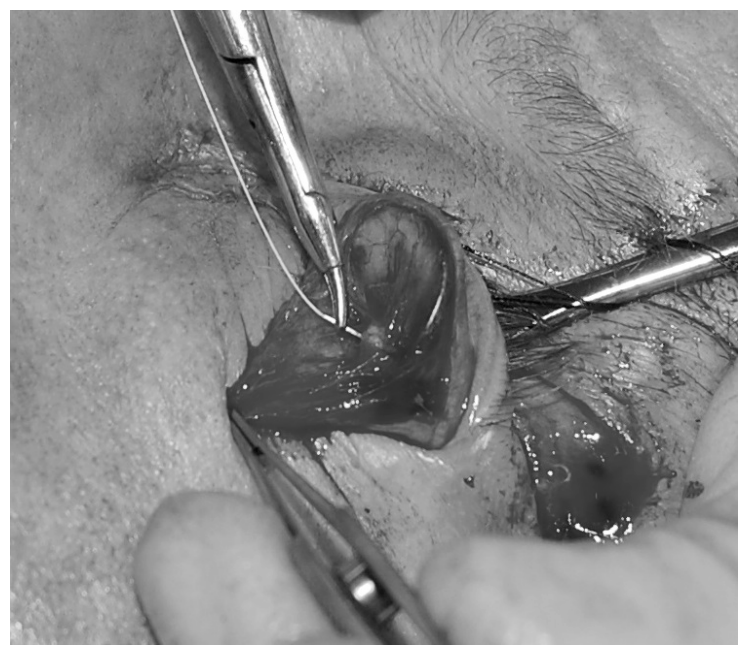

(B)

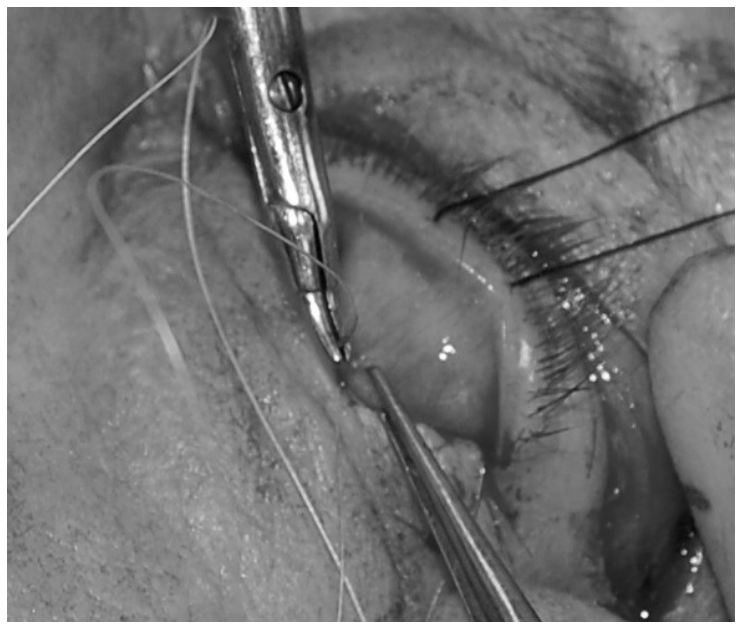

(C)

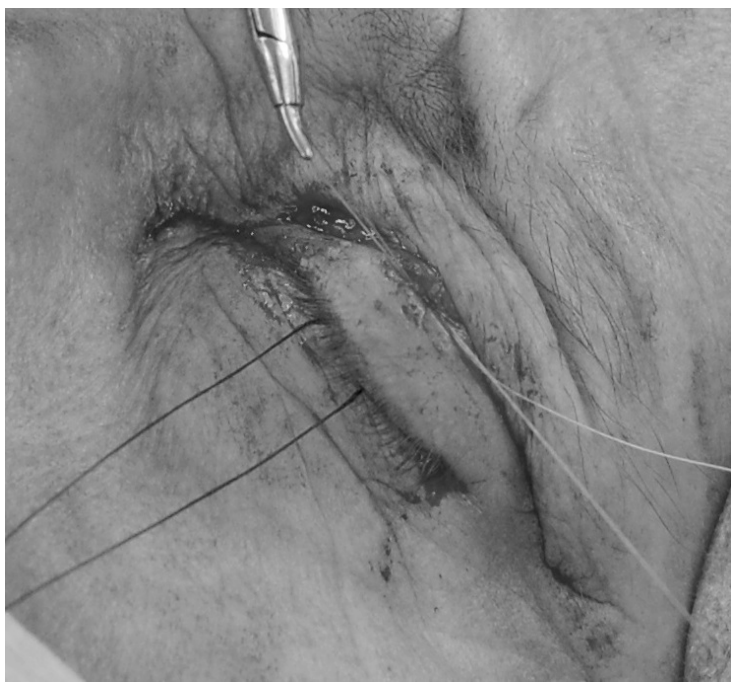

Fig. (1). Surgical Technique. A double armed 5-0 vicryl ${ }^{\mathbb{R}}$ suture is placed centrally through the posterior belly of the white-line (A), in a forehand manner and is then passed through the conjunctival surface of the tarsal plate, $1 \mathrm{~mm}$ below its superior border (B), and then through to the skin. The eyelid height and contour is assessed after tying this first suture in a bow $(\mathbf{C})$. muscle fibres, and becomes confluent with the lower one third of the tarsal plate and subcutaneous tissue. We have found that erroneous placement of sutures into the orbital septum, which can occasionally appear as the white line, leads to an undercorrection of ptosis. After further dissection the healthier white sheet can be found and effective advancement can be achieved by placing the sutures into this posterior surface of the levator aponeurosis.

\section{DISCUSSION}

Our technique is similar to that reported by Khooshabeh $[12,13,16]$ in so far as it involves initial dissection of the composite flap of Müller's muscle and conjunctiva with direct visualization of the tissues concerned. However once the distal border of the levator aponeourosis is identified (the "white-line"), double armed sutures are passed through this white-line and then through the superior tarsus and skin. Only the aponeurosis is therefore advanced and neither Müller's muscle or conjunctiva are excised. It can also be easily combined with a blepharoplasty.

Many believe that if the eyelid fails to respond to the phenylephrine test an alternative to the MMCR such as external levator resection should be performed. However posterior approach white-line advancement can be used irrespective of the phenylephrine test as it involves advancement of the levator aponeurosis rather than MMCR. Some believe that excision of the conjunctiva may damage the accessory lacrimal glands of Wolfring, situated in the upper tarsal border, which may aggravate dry eyes [12] Others [21] have found no effect on tear production. However with no conjunctival excision this potential risk can be avoided.

Levator advancement through a posterior approach has been previously reported. In 1979, Collin et al. [22] reported a technique of reinserting the aponeurosis via a posterior approach. This involved exposing the anterior surface of levator aponeurosis by dissection of the orbital septum and retraction of the preaponeurotic fat pad, effectively converting a posterior approach to the familiar anatomical view of an anterior approach ptosis repair. The initial posterior incision was recommended to be made $2 \mathrm{~mm}$ below the superior border of the tarsus in order to enter a tissue plane anterior to Müller's muscle. Consequently, although conjunctiva was largely preserved, the distal $2 \mathrm{~mm}$ of Müller's muscle and $2 \mathrm{~mm}$ of superior tarsus were excised. Any degree of tarsal resection will further contribute to eyelid elevation regardless of muscle resection or levator advancement.

Ichinose et al. [23] more recently used a similar technique to Collin et al. without resecting Müller's muscle. The initial incision was $1 \mathrm{~mm}$ below the superior border of the tarsus. Their technique also involved dissection of the orbital septum and exposure of the anterior surface of the levator aponeurosis. The aponeurosis was advanced down and sutured to the frontosuperior part of the tarsus. In addition Müller's muscle was reattached to the superior edge of the tarsal plate. Both of these techniques involved dissection of the levator aponeurosis anteriorly from its surrounding structures before its advancement.

Our technique differs in several ways. We do not dissect septum to expose the anterior surface of levator aponeurosis. 
We simply pass the sutures through the distal border of the levator aponeurosis. Our initial incision is just above the superior border of the tarsus as opposed to below, avoiding any excision of tarsus. There is no excision of Müller's muscle or conjunctiva and the Müller's-conjunctiva composite flap we create is simply replaced without the need for resuturing.

External levator advancement for blepharoptosis is an effective procedure in establishing good eyelid position, with reported success rates of 70\% to more than $95 \%$ [24-27]. Reports of Müller's muscle resection would suggest a higher success rate compared to that for anterior approach levator advancement. Putterman [28] published a study showing 90 $\%$ of eyelids achieving within $1.5 \mathrm{~mm}$ symmetry of the fellow eye. However these results were for predominately mild phenylephrine positive ptosis and if applied to more stringent criteria (defined as a postoperative MRD of $\geq 2 \mathrm{~mm}$ and $\leq 4.5 \mathrm{~mm}$, inter-eyelid height asymmetry of $\leq 1 \mathrm{~mm}$, and satisfactory eyelid contour), would result in a success rate of $75 \%$. Dresner [11] reported $85 \%$ of eyelids being within 0.5 $\mathrm{mm}$ symmetry. Lake et al. [12] describing their open sky technique in phenylephrine positive patients, showed $98 \%$ were within $1 \mathrm{~mm}$ symmetry with the fellow eye. In their second study [16] looking at phenylephrine negative patients all were within $0.5 \mathrm{~mm}$ symmetry of the fellow eye. Our Müller's muscle-conjunctival sparing technique results in a predictable degree of lid height and contour. In our series there was an $87 \%$ success rate and all patients had a good contour. We feel it can be used in the majority of ptosis patients with moderate to good levator function.

The transconjunctival route still has a role in Müller's ptosis surgery. Although previously reserved for those with mild phenylephrine positive cases, newer techniques of posterior approach levator advancement are now an option in the majority of aponeurotic ptosis with moderate to good levator function irrespective of the response to phenylephrine.

\section{CONFLICT OF INTEREST}

The authors have no conflict of interest or financial interest related to this manuscript.

\section{REFERENCES}

[1] Blaskovicz L. A new operation for ptosis with shortening of the levator and tarsus. Arch Ophthalmol 1923; 52: 563-73.

[2] Blaskovicz L. Treatment of ptosis. The formation of a fold in the eyelid and resection of the levator and tarsus. Arch Ophthalmol $1929 ; 1: 672-80$

[3] Agatston SA. Resection of Levator Palpebrae muscle by the conjunctival route for ptosis. Arch Ophthalmol 1942; 27: 994-6.

[4] Berke RN. A simplified Blaskovics operation for blepharoptosis; results in ninety-one operations. AMA Arch Ophthalmol 1952; 48: 460-95.
[5] Iliff CE. A simplified ptosis operation. Am J Ophthalmol 1954; 37 : 529-33.

[6] Fasanella RM, Servat J. Levator resection for minimal ptosis: another simplified operation. Arch Ophthalmol 1961; 5: 493.

[7] Fox SA. A modified Fasanella-Servat procedure for ptosis. Arch Ophthalmol 1975; 93: 639-40.

[8] Beard C. Blepharoptosis repair by modified fasanella servat operation. Am J Ophthalmol 1970; 9: 850-7.

[9] Putterman AM, Urist MJ. Müller muscle-conjunctiva resection. Arch Ophthalmol 1975; 93: 619-23.

[10] Weinstein GS, Buerger GF Jr. Modification of the Müller's muscleconjunctival resection operation for blepharoptosis. Am J Ophthalmol 1982; 93: 647-51.

[11] Dresner SC. Further modifications of the Müller's muscleconjunctival resection procedure for blepharoptosis. Ophthal Plast Reconstr Surg 1991; 7: 114-22.

[12] Lake S, Mohammad-Ali FH, Khooshabeh R. Open sky Müller's muscle-conjunctiva resection for ptosis surgery. Eye 2003; 17: 1008-12.

[13] Khooshabeh R, Baldwin HC. Isolated Müller's muscle resection for the correction of blepharoptosis. Eye 2008; 22: 267-72.

[14] Buchman G, Jakobiec FA, Hyde K, et al. Success of Fasanella Servat operation independent of Müller's smooth muscle excision. Ophthalmology 1989; 96: 413-8.

[15] Perry JD, Kadakia A, Foster JA, A new algorithm for ptosis repair using conjunctival Müllerectomy with or without tarsectomy. Ophthal Plast Reconstr Surg 2002; 18: 426-9.

[16] Baldwin HC, Bhagey J, Khooshabeh R. Open sky Müller muscleconjunctival resection in phenylephrine test-negative blepharoptosis patients. Ophthal Plast Reconstr Surg 2005; 21: 276-80.

[17] Mercandetti M, Putterman AM, Cohen ME, et al. Internal levator advancement by Müller's muscle-conjunctival resection. Arch Facial Plast Surg 2001; 3: 104-10.

[18] Buckman G, Jakobiec FA, Hyde K, et al. Success of the FasanellaServat operation independent of Müller's smooth muscle excision. Ophthalmology 1989; 96: 413-8.

[19] Kakizaki H, Zako M, Nakano T, et al. The levator aponeurosis consists of two layers that include smooth muscle. Ophthal Plast Reconstr Surg 2005; 21: 379-82.

[20] Kakizaki H, Madge SN, Malhotra R, Selva D. The levator aponeurosis contains smooth muscle fibers: new findings in Caucasians. Ophthal Plast Reconstr Surg 2009; 25: 267-9.

[21] Dailey RA, Saulny SM, Sullivan SA. Müller muscle conjunctival resection effect on tear production. Ophthal Plast Reconstr Surg 2002; 6: 421-5.

[22] Collin JR. A ptosis repair of aponeurotic defects by the posterior approach. Br J Ophthalmol 1979; 63: 586-90.

[23] Ichinose A, Tahara S. Transconjunctival levator aponeurotic repair without resection of Müller's muscle. Aesthetic Plast Surg 2007; 31: 279-84.

[24] Anderson RL, Dixon RS. Aponeurotic ptosis surgery. Arch Ophthalmol 1979; 97: 1123-8.

[25] Older JJ. Levator aponeurosis surgery for the correction of acquired ptosis: analysis of 113 procedures. Ophthalmology 1983; 90: 10569.

[26] Berlin AJ, Vestal KP. Levator aponeurosis surgery: a retrospective review. Ophthalmology 1989; 96:1033-6.

[27] McCulley TJ, Kersten RC, Kulwin DR, Feuer WJ. Outcome and influencing factors of external levator palpebrae superioris aponeurosis advancement for blepharoptosis. Ophthal Plast Reconstr Surg 2003; 19: 388-93.

[28] Putterman AM, Fett DR. Müller's muscle in the treatment of upper eyelid ptosis: a ten-year study. Ophthalmic Surg 1986; 17: 354-60. 